\title{
COVID-19 in allogeneic stem cell transplant: high false-negative probability and role of CRISPR and convalescent plasma
}

\author{
Alex Niu ${ }^{1}$ April McDougal ${ }^{2} \cdot$ Bo Ning $^{3} \cdot$ Firas Safa ${ }^{1} \cdot$ Alfred Luk $^{2} \cdot$ David M. Mushatt $^{2} \cdot$ Adeem Nachabe $^{4}$. \\ Kevin J. Zwezdaryk ${ }^{5}$. James Robinson ${ }^{6} \cdot$ Tim Peterson $^{7}$. Francisco Socola ${ }^{1} \cdot$ Hana Safah $^{1} \cdot$ Tony Hu $^{3}$. \\ Nakhle S. Saba ${ }^{1}{ }^{1}$
}

Received: 19 May 2020 / Revised: 26 May 2020 / Accepted: 3 June 2020 / Published online: 15 June 2020

(c) Springer Nature Limited 2020

\section{To the Editor:}

While immunosuppression may be beneficial in later phases of COVID-19, it can delay virus clearance if applied too early. In immunocompromised patients, such as allogeneic stem cell transplant (ASCT) recipients, testing strategies, disease outcomes, and choice of therapy remain largely unknown.

Due to concerns of high rates of false-negative results with RT-PCR based assays, the American Society for Transplantation and Cellular Therapy (ASTCT) recommends repeat SARS-CoV-2 testing in ASCT patients if initial testing is negative [1]. Bronchoalveolar lavage (BAL)

These authors contributed equally: Alex Niu, April McDougal

Supplementary information The online version of this article (https:// doi.org/10.1038/s41409-020-0972-8) contains supplementary material, which is available to authorized users.

Nakhle S. Saba

nsaba@tulane.edu

1 Section of Hematology and Medical Oncology, Deming Department of Medicine, Tulane University, New Orleans, LA, USA

2 Section of Infectious Diseases, Deming Department of Medicine, Tulane University, New Orleans, LA, USA

3 Department of Biochemistry \& Molecular Biology, Tulane University, New Orleans, LA, USA

4 Louisiana State University School of Medicine, New Orleans, LA, USA

5 Department of Microbiology and Immunology, Tulane University, New Orleans, LA, USA

6 Section of Pediatric Infectious Disease, Department of Pediatrics, Tulane University, New Orleans, LA, USA

7 Department of Pathology and Laboratory Medicine, Tulane University, New Orleans, LA, USA might improve testing sensitivity, but is not routinely performed due to operator safety concerns [1, 2]. Therefore, if upper respiratory tract RT-PCR testing is negative and clinical suspicion remains high, diagnosis may rely on pulmonary imaging and symptomatology [2]. In addition, while the viral genome has been detected in other bodily sources, such as blood, feces, and sputum, its clinical significance remains unclear and has not been studied in the setting of ASCT [2]. With their goal to improve sensitivity, the US Food and Drug Administration recently issued an Emergency Use Authorization to a highly sensitive clustered regularly interspaced short palindromic repeats (CRISPR)-based qualitative COVID-19 assay [3].

Herein, we report two cases of COVID-19 infection in ASCT recipients using CRISPR diagnostics, followed by successful treatment with COVID-19 convalescent plasma (CCP).

Case 1: A 53-year-old female with B-cell acute lymphoblastic leukemia, status post haploidentical ASCT (Supplementary Table 1), presented on day +157 post ASCT with fever, shortness of breath, and cough. Her case is complicated by steroid-dependent chronic graft-versushost disease (cGVHD) of the skin and mouth, currently responding to weekly intravenous rituximab. A chest computed tomography (CT) scan revealed ground glass opacities suggestive of COVID-19 (Fig. 1a). Both nasal and nasopharyngeal RT-PCR swab tests (Abbott and Roche) were negative for SARS-CoV-2. She was started on ceftriaxone, azithromycin, and vancomycin for pneumonia. Intravenous immunoglobulin (IVIG) was administered for hypogammaglobulinemia. Over the proceeding 48 hours, her respiratory status worsened, requiring oxygen at $4 \mathrm{~L} / \mathrm{min}$. Due to continued suspicion for COVID-19, investigational RT-PCR/CRISPR technology was performed as we have previously described (supplementary methods) [4], on nasal swab, saliva, blood, and plasma samples, and surprisingly tested positive for SARS-CoV-2 

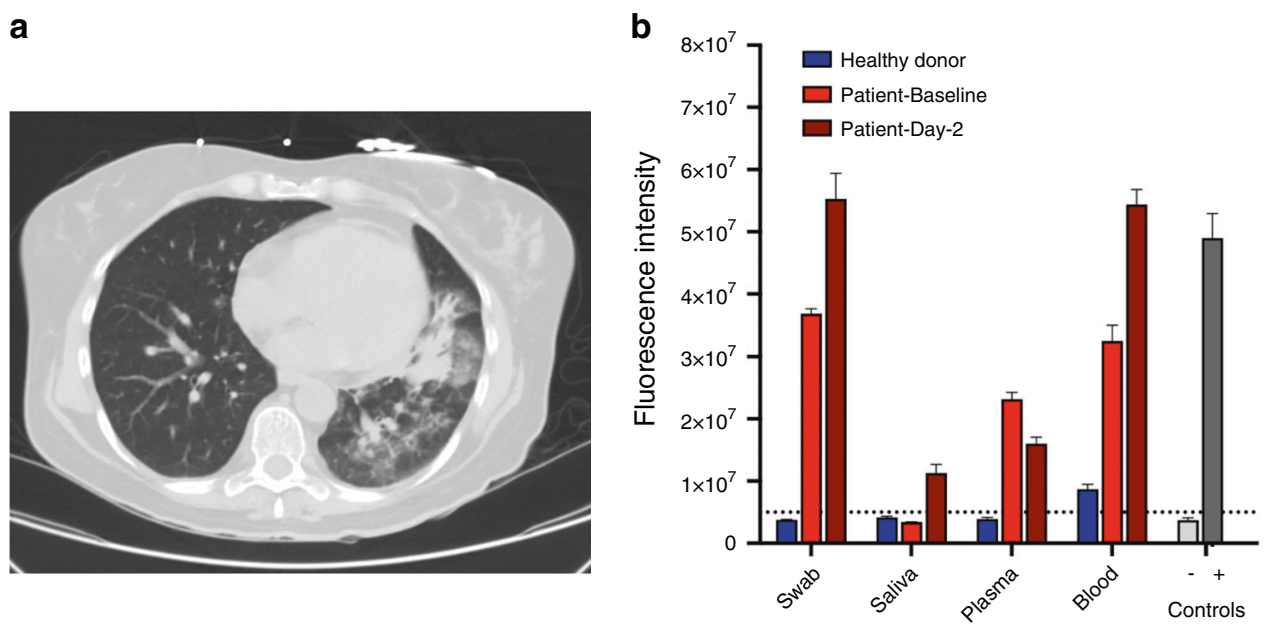

C

Fig. 1 Diagnostic workup for case 1 and 2. a Transverse CT scan showing lung infiltrates suspicious for COVID-19 disease for case 1 . b CRISPR analysis testing for the presence of the viral genome in the shown sample of case 1 . We used genomic RNA extracted from heatinactivated SARS-CoV-2 (NR-52347, BEI) as positive control, and human RNA isolated from HEK293T cells as negative control. Each

RNA (Fig. 1b). One unit (200 ml) of CCP was given on hospital day 4 and day 13 . Within a day of receiving her first transfusion of CCP, she reported improvement in shortness of breath and cough, had fever resolution, and decreasing oxygen requirements. In addition, a significant decrease in C-reactive protein and procalcitonin was also seen (Supplementary table 1). The patient was discharged home without oxygen on hospital day 15 .

Case 2: A 67-year-old male with a history of ASCT for high-risk acute myeloid leukemia (Table S1), presented on day +319 post ASCT with shortness of breath, cough, and a worsening skin rash. His transplant was complicated by steroid refractory cGVHD of the skin and lungs requiring treatment with the Bruton's tyrosine kinase (BTK) inhibitor ibrutinib in addition to prednisone and rituximab. A chest CT revealed ill-defined peribronchovascular opacities raising concern for COVID-19. However, he tested negative for SARS-CoV-2 RNA via nasopharyngeal RT-PCR (Roche). He was started on ceftriaxone and doxycycline and received IVIG for hypogammaglobulinemia. On hospital day 11, his respiratory culture grew multidrug resistant Stenotrophomonas maltophilia, and intravenous trimethoprim-sulfamethoxazole was started. The patient's condition

progressively worsened to acute respiratory distress requiring mechanical ventilation. Bronchoscopy was performed, but no definitive diagnosis was made. Repeat SARS-CoV-2 RT-PCR was performed on a nasal swab and BAL specimen, but both were again negative. Despite aggressive antimicrobial therapy and supportive care, the patient developed multiorgan failure and died on hospital day 36 (+354 post ASCT). An autopsy was not performed due experiment was run in triplicate. Values that are $3 \mathrm{SD}$ above the negative control mean were considered positive. The level of fluorescence does not reflect viral load, this is a qualitative test. c CRISPR analysis testing for the presence of the viral genome in the shown sample of case 2 tested as in $\mathbf{b}$.

to concern for possible COVID-19 and the risk it posed to the staff. Shortly thereafter, RT-PCR/CRISPR was performed on a blood sample collected on hospital day 36 for clinical purposes and demonstrated strong detection of SARS-CoV-2 RNA (Fig. 1c).

Here, we present two cases of ASCT recipients who presented with respiratory illnesses, initially testing negative for SARS-CoV-2 with conventional RT-PCR, then positive with the more sensitive RT-PCR/CRISPR technique. One patient was diagnosed with COVID-19 early in her course and was immediately treated with CCP and survived, while the other did not receive COVID-19 directed therapy and expired. Our findings suggest that ASCT recipients with negative nasopharyngeal SARSCoV-2 RT-PCR, but evidence of lower respiratory tract disease, might indeed have COVID-19 that can be detected using a CRISPR-based platform. This challenges the established algorithm for evaluation and treatment of suspected/confirmed COVID-19 in ASCT recipients and illustrates the need for more sensitive tests. Prompt diagnosis is essential in all patients, particularly in immunocompromised hosts, as earlier treatment will likely improve outcomes. Antiviral agents, such as remdesivir, have recently been shown to shorten time to clinical improvement particularly with earlier initiation [5, 6]. Similarly in the SARS-CoV-1 2003 epidemic, the clinical benefit of convalescent plasma was more significant if given within the first 14 days of illness [7].

Our cases tested positive for SARS-CoV-2 in samples other than a nasopharyngeal source. This poses the question of whether patients with objective findings consistent with COVID-19 and a negative nasopharyngeal RT-PCR assay 
would benefit from testing of other tissue types [2]. In the particular example of ASCT, the ASTCT suggests that a negative nasopharyngeal RT-PCR test does not rule out COVID-19 in patients with lower respiratory tract symptoms, as the virus might be detectable only in the lower airways. While safety concerns with performing bronchoscopy remain high with this infection, and COVID-19specific treatments depend on securing a positive test, it may be beneficial to pursue diagnosis with other tissue sources, such as whole blood or plasma.

As our report demonstrates, ASCT recipients are at risk of developing more severe complications from COVID-19. T-cell lymphopenia has been correlated with higher risk of in-hospital death and severity of COVID-19 infection [8]. Indeed, our patients have low baseline T-lymphocyte counts further decreased with cGVHD therapy with steroids, tacrolimus, and ibrutinib. Similarly, SARS-CoV-2 may cause a decrease in B-lymphocytes early in the disease course, thus hindering the ability to produce antibodies [9]. In our patients, the ability to develop memory B-cells and plasma cells for antibody production is already impeded by steroids and rituximab. Thus, passive immunity support with CCP is required to deliver the SARS-CoV-2 neutralizing antibodies needed to clear the infection.

It may be most appropriate to preemptively treat with investigational therapeutics in patients where high clinical suspicion exists but test negative. In our case, we elected to treat with CCP; however, combination therapy could be considered. Interim remdesivir data have shown clinical benefit in symptom duration and overall survival [5]. The addition of CCP should be considered adjunctively moving forward in this high-risk population due to a jeopardized active immunity. Our patient who received CCP significantly improved, which is consistent with other CCP reports [10]. However, as ASCT recipients on immunosuppression are less likely to generate adequate antibodies, multiple transfusions of CCP might be needed as in case 1 .

There were several differences between our two case patients (Table S1). Notably the clinical presentation, with case 1 presenting acutely, while case 2 had a progressive clinical course. Both patients were taking steroids; however, case 2 was on ibrutinib. Intriguingly, the BTK inhibitors, ibrutinib and acalabrutinib, are being investigated for their ability to reduce COVID-19 induced respiratory distress $[11,12]$. This is notable as case 2 intermittently continued ibrutinib due to his protracted illness and had a more insidious COVID-19 infection with slower progression to respiratory failure.

In conclusion, early diagnosis and treatment of COVID19 is crucial in ASCT recipients, and evaluation regarding the use of other tissue sources for detection of SARS-CoV-2 along with multimodality therapy is required in the continual evolution of this pandemic.
Acknowledgements We thank our patients for participating and donating the tissue samples to make this research possible. We also thank the Louisiana Cancer Research Center biospecimen core for their help in consenting and sample collection process.

Author contributions ANiu, AM, and NSS collected and analyzed data and wrote the manuscript. TH and BN performed the RT-PCR/ CRISPR assay. All other authors contributed to treatment, reviewing, editing, and writing of the manuscript.

\section{Compliance with ethical standards}

Conflict of interest NSS: Advisory board and speaker bureau for ibrutinib. The other authors declare no conflict of interest.

Publisher's note Springer Nature remains neutral with regard to jurisdictional claims in published maps and institutional affiliations.

\section{References}

1. COVID-19 Resource Community. American Society for Transplantation and Cellular Therapy. COVID-19 Resource Community; 2020. https://www.astct.org/communities/public-home? CommunityKey $=\mathrm{d} 3949 \mathrm{~d} 84-3440-45 \mathrm{f} 4-8142-90 \mathrm{ea} 05 \mathrm{adb} 0 \mathrm{e}$.

2. Wang W, Xu Y, Gao R, Lu R, Han K, Wu G, et al. Detection of SARS-CoV-2 in Different Types of Clinical Specimens. JAMA. 2020;323:1843-4.

3. Jennings K. FDA Authorizes First-Ever Crispr Application For COVID-19 Coronavirus Test Forbes2020 [updated 2020 May 7]. Available from: https://www.forbes.com/sites/katiejennings/2020/ 05/07/fda-authorizes-first-ever-crispr-application-for-covid-19coronavirus-test/\#37d3bbb91708.

4. Huang Z, Tian D, Liu Y, Lin Z, Lyon CJ, Lai W, et al. Ultrasensitive and high-throughput CRISPR-p owered COVID-19 diagnosis. Biosens Bioelectron. 2020;164:112316.

5. Grein J, Ohmagari N, Shin D, Diaz G, Asperges E, Castagna A, et al. Compassionate Use of Remdesivir for Patients with Severe Covid-19. N Engl J Med. 2020;382:2327-36.

6. Beigel JH, Tomashek KM, Dodd LE, Mehta AK, Zingman BS, Kalil AC, et al. Remdesivir for the Treatment of Covid-19 Preliminary Report. N Engl J Med. 2020.

7. Cheng Y, Wong R, Soo YOY, Wong WS, Lee CK, Ng MHL, et al. Use of convalescent plasma therapy in SARS patients in Hong Kong. Eur J Clin Microbiol Infect Dis. 2005;24:44-6.

8. Xu B, Fan C-Y, Wang A-L, Zou Y-L, Yu Y-H, He C, et al. Suppressed T cell-mediated immunity in patients with COVID-19: a clinical retrospective study in Wuhan, China. J Infect. 2020; S0163-4453:30223-1.

9. Lin L, Lu L, Cao W, Li T. Hypothesis for potential pathogenesis of SARS-CoV-2 infection-a review of immune changes in patients with viral pneumonia. Emerg Microbes Infect. 2020;9:727-32.

10. Shen C, Wang Z, Zhao F, Yang Y, Li J, Yuan J, et al. Treatment of 5 critically Ill patients with COVID-19 with convalescent plasma. JAMA. 2020;323:1582-9.

11. ClinicalTrials.gov [Internet]. Bethesda (MD): National Library of Medicine (US). 2000 Feb 29 - . Identifier NCT04346199, Acalabrutinib Study With Best Supportive Care Versus Best Supportive Care in Subjects Hospitalized With COVID-19; 2020 [cited 2020 May 4]. https://ClinicalTrials.gov/show/NCT04346199.

12. Treon SP, Castillo JJ, Skarbnik AP, Soumerai JD, Ghobrial IM, Guerrera ML, et al. The BTK inhibitor ibrutinib may protect against pulmonary injury in COVID-19-infected patients. Blood. 2020;1351:1912-5. 